\title{
Chapter 4 \\ Deriving Experience Curves \\ and Implementing Technological \\ Learning in Energy System Models
}

\author{
Atse Louwen and Martin Junginger
}

\begin{abstract}
Technological learning encompasses a variety of mechanisms by which technologies improve and decrease in costs. Experience curves are commonly used to analyze and explicitly quantify technological learning. This chapter presents the history and basic methodology of experience curves, and discusses the implementation of experience curves in energy system and sectoral energy models. Several key results of the REFLEX project with respect to state-of-the-art experience curves, and the implementation of experience curves in the REFLEX Energy Modeling System are highlighted. Finally, a set of key lessons learned in the REFLEX project are presented, discussing both methodological issues of experience curves as well as key issues with regard to the implementation of experience curves in different types of energy system and sectoral energy models.
\end{abstract}

\subsection{Introduction}

\subsubsection{History and Concept}

Within the REFLEX project, a large effort was made to include the effects of technological learning in the different energy and transport models that are used within the project. Here, technological learning is considered as a term that encompasses a variety of mechanisms by which technologies can improve, in relation to production costs, efficiency, quality, etc. It includes mechanisms like learning-by-doing, learning-by-searching (R\&D), and upscaling. One of the most prominent methods to analyze and quantify technological learning is the so-called 'experience curve.'

The experience curve describes an empirical relationship between cumulative production of a technology and its unit costs. It was developed in the form considered

\footnotetext{
A. Louwen $\cdot$ M. Junginger $(\bowtie)$

Copernicus Institute of Sustainable Development, Utrecht University, Utrecht, The Netherlands e-mail: H.M.Junginger@uu.nl
}

\section{A. Louwen}

Institute of Renewable Energy, Eurac Research, Bolzano, Italy 
here by the Boston Consulting Group, building on work that originated from the aircraft building Curtiss-Wright Corporation (Wright 1936). Wright analyzed the labor costs for each airplane, and found that they decreased for each additional airplane that was manufactured. He showed that the costs followed the following relation:

$$
F=N^{x}
$$

Here, $F$ is the variation of production cost as a function of the cumulative quantity of airplanes $N$, and $x$ is the factor that gives the speed with which the labor costs declined. The reciprocal of this formula gives the decline in labor costs with increasing $N$. With a value of $x=0.322$ in Eq. (4.1), labor costs declined with $20 \%$ every time the cumulative quantity of airplanes produced doubled. This relation, now referred to as the learning curve, was later revisited by different researchers, until the Boston Consulting Group (1970) developed the experience curve, which aimed to include total costs of production (not just labor) and would represent a whole industry of a technology, rather than a single company. They gave the equation the following form:

$$
C=C_{1} \cdot N^{b}
$$

Here the unit costs of a technology $C$ are a function of the cumulative production $N$, the experience curve parameter $b$ and the costs of the first unit, $C_{1}$. With a value of $b=-0.322$, production costs would again decline with $20 \%$ for every doubling of $N$. This also gave rise to two terms associated with the experience curve parameter $b$, namely the learning rate (LR) and progress ratio (PR), with:

$$
\begin{gathered}
L R=1-2^{b} \\
P R=2^{b}
\end{gathered}
$$

The learning rate $L R$ gives the percentage reduction in costs for every doubling of cumulative production $N$, while the progress ratio $P R$, is $80 \%$ if the learning rate is $20 \%$. The experience curve of Eq. (4.2) is now called the one-factor experience curve (OFEC), as it includes only the cumulative production as a factor explaining costs reductions. Further development of the concept has resulted in the two-factor experience curve (TFEC), which includes a parameter that describes R\&D (using R\&D expenditures or patent applications as a proxy) and multi-factor experience curves (MFEC) which could include a variety of additional parameters, such as input material prices. As the aim of the REFLEX project is to include technological progress in energy modeling systems, and these models generally do not produce all inputs required for two- or multi-factor experience curves (Louwen et al. 2018), the focus is here on the one-factor experience curves. Figure 4.1 shows an example 


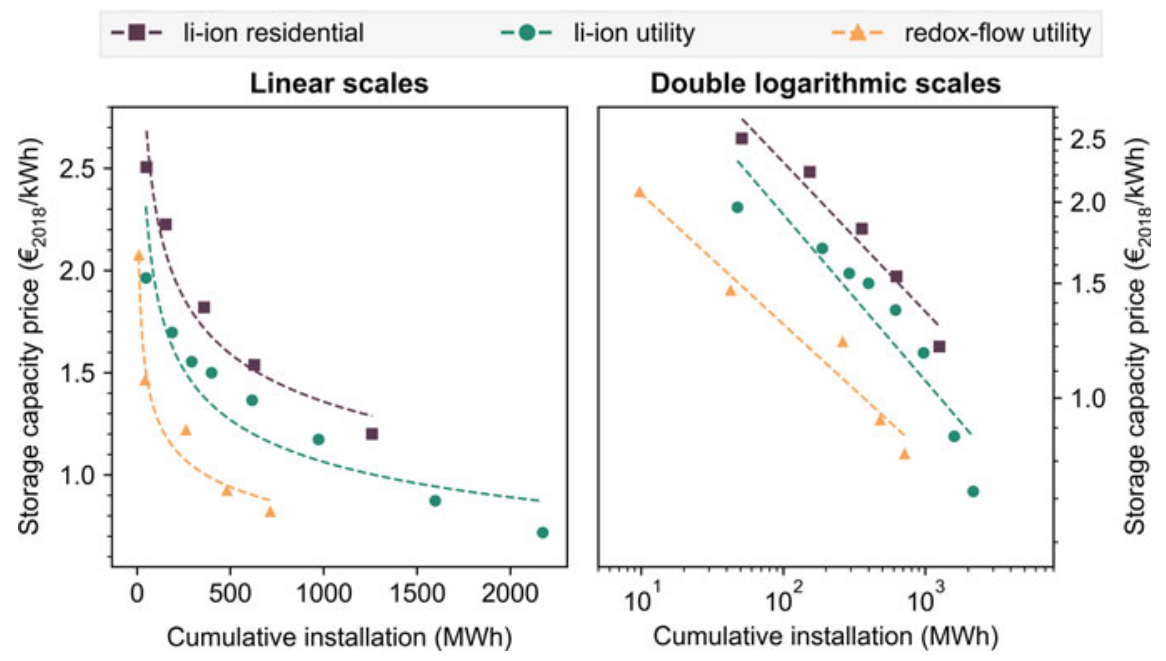

Fig. 4.1 Example of experience curves in linear (left) and double-logarithmic (right) scales. Data for battery storage systems according to Schmidt et al. (2017)

of experience curves for battery storage systems on linear and double-logarithmic scales.

Although not without criticisms, the OFEC is one of the few methods that use an empirical relationship between production costs and cumulative production as a means to forecast future costs of technologies (Junginger and Louwen 2020). In modeling activities, it allows for representing developments in the market diffusion of technologies and gives cost trajectories of the respective technologies over the timeframe of the modeling. In Sect. 4.3, the model implementation of experience curves is discussed in more detail.

\subsubsection{Key Applications of Experience Curves}

The experience curve has two main applications: as a tool in the design and assessment of policy measures, and as a tool with which future cost trajectories can be determined in energy and integrated modeling activities.

For policymakers, the experience curve can be a valuable means to track the cost developments of for instance renewable alternatives for electricity generation, but also to design and assess policy measures that aim to bring down the costs of these technologies. By deriving an experience curve from empirical cost data, a deployment trajectory can be defined, showing how much additional cumulative production is required for the technology to reach a certain competitive cost level. Then, by taking the integral of the experience curve, the so-called 'learning investments' can be derived (Louwen and Subtil Lacerda 2020). These learning investments, which are 


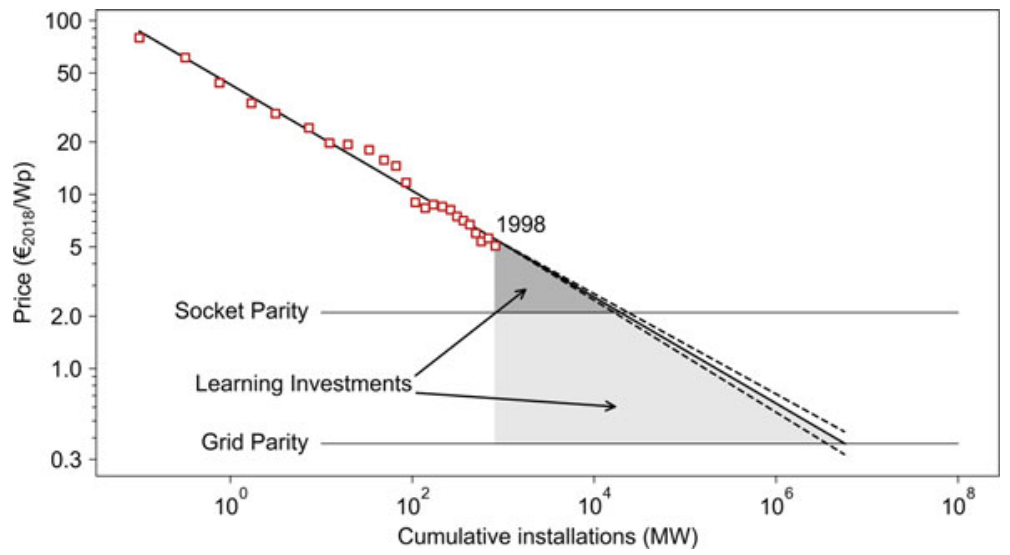

Fig. 4.2 Example of a visualization of the required learning investments to reach a certain level of competitiveness (Source Figure taken from Louwen and Subtil Lacerda [2020])

the total cost, minus the competitive cost share, can be considered as the investment that needs to be incentivized through policy measures before the technology becomes competitive without subsidies. In Fig. 4.2 an example is shown that visualizes these learning investments.

A good example of the effect of such policies on driving prices down the experience curve is found for the solar photovoltaic (PV) industry. Due to incentive schemes in several European markets, especially Germany, the demand for PV systems grew rapidly in the past decades. As a result, cumulative production of PV systems increased, and system costs declined. At a later stage, many other local PV markets started growing in response to declining costs, and especially in China installed PV system capacity grew rapidly, resulting in that country now having the largest amount of installed PV system capacity. As a result of the rapid decline in PV system costs, PV generated electricity is now competitive with fossil fuel alternatives in ever more countries (SolarPower Europe 2019).

Another main application of the experience curve lies in energy and integrated modeling activities. Since the main aim of energy and integrated modeling is to give forecasts of future energy and climate systems, a key aspect of this modeling is to be able to model the future costs of energy system technologies. Usually, these future costs could be taken from time-based exogenous cost estimations, but by using experience curves, models can endogenously determine cost trajectories for technologies in response to and in a feedback loop with their modeled market diffusion trajectories. The fact that the experience curve is based on empirical data makes it very attractive in modeling and offers a very simple model formulation of costs vs. cumulative production.

By using experience curves, modelers thus have an evidence-based method to calculate future technology investment costs, and as such can model future energy systems while considering the feedback loop between technology cost and market 
diffusion. This gives both researchers and policymakers a tool with which they can design pathways toward future low-carbon energy systems, and assess the requirements and effects of policy measures that aim to increase market diffusion of technologies or to achieve certain climate change mitigation targets.

\subsubsection{Key Issues and Drawbacks of Experience Curves}

An overview of several issues and drawbacks of (using) experience curves is given in Junginger et al. (2010) and Junginger and Louwen (2020). Below some key issues will be discussed, related to experience curve parameter uncertainty, system boundaries and functional units, and causality.

\subsubsection{Experience Curve Parameter Uncertainty}

A common issue with deriving curve parameters through regression of empirical datasets, is the uncertainty in the derived parameters. For experience curves this is no different. Cost projections made with experience curves are thus extremely sensitive to uncertainties. However, uncertainty about the value of the experience curve parameters is the result of two issues observed in experience curve studies.

First, a study by Nemet (2009) shows that the learning rate derived from subsets of the complete dataset is not constant, and as a result, a calculation of required learning investments as described in Sect. 4.1.2 can vary wildly. This would suggest that the recommendation is to use datasets that have as long a timeframe as possible. Given the fact that datasets for novel technologies like electricity storage systems are however quite small (Kittner et al. 2017; Schmidt et al. 2017), this means rigorous uncertainty and sensitivity analysis of experience curve parameters is required.

Second, a common problem with (non-)linear regression is that with large errors between measured and modeled data, the uncertainty of the derived experience curve parameters can also be substantial. When using these parameters for cost extrapolations, it is recommended to take into account this uncertainty, e.g., by presenting the confidence interval of extrapolations, or by using a stochastic model formulation of parameter uncertainty (van Sark et al. 2010).

\subsubsection{System Boundaries and Functional Units}

The definition of system boundaries, and the choice of the functional unit affects the value of the derived experience curves, and to a certain extent determines whether any reasonable experience curve can be derived at all. If technologies are aggregates of multiple components, it is fair to assume these components each have specific potential for cost reduction, and thus would have different learning rates. Using experience 
curves for the aggregate system would in that case overestimate future cost reductions, and a recommendation is to use either component-based experience curves (Junginger and Louwen 2020), or to devise some form of a piece-wise experience curve (Kittner et al. 2017).

A similar system boundary issue results from technologies that are in fact heterogeneous, but are normally considered as a single technology such as lithium battery electricity storage systems, which vary in materials used and their designs.

When deriving experience curves, it is important to determine the appropriate functional unit. As recent studies for e.g., wind power show (Williams et al. 2017), unit capacity prices of wind turbines and systems have gone up and down the past decade, making derived single factor experience curves for unit capacity cost nearly useless. However, many developments in the wind power industry have focused on reducing levelized cost of electricity, rather than unit capacity prices. Hence, a more useful experience curve can be derived for wind LCOE. Furthermore, capacity prices are affected by subsidies as producers take these into account to determine acceptable market prices.

\subsubsection{Explanatory Value of Single-Factor Experience Curves}

As is often mentioned, the single-factor experience curve on its own offers no explanation of the observed cost reductions, and cannot prove any causality between cumulative production and cost developments (Yeh and Rubin 2012). With multi-factor curves, this issue can be partly alleviated, although different learning mechanisms are often difficult to separate, as the proxy data used to analyze them (such as cumulative production and patent applications) often is highly correlated in growing industries. Experience curve studies should thus ideally be accompanied with a thorough examination of the reasons behind the observed cost developments.

\subsection{Data Collection and Derivation of Experience Curves}

In order to derive the parameters of Eq. (4.2), and the learning rate and progress ratio, historical data needs to be collected on cumulative production and cost developments of the studied technology. Commonly, especially for more mature technologies, cumulative production figures are easily collected, sometimes calculated based on cumulative sales or cumulative installation data as proxies. Productions costs of technologies are generally much more difficult to find however, and therefore many experience curves studies use market prices as proxy data. For mature technologies in a stable market, it is often assumed that market prices and production costs decline at an equal rate, but in early market diffusion stages, subsidized markets or during supply/demand imbalances, there can be significant differences between market prices and production costs (Boston Consulting Group 1970). Still, out of necessity it is often unavoidable to use market price data as cost data is not 
publicly available. Furthermore, this can also be justified as investments decisions in technologies are usually made based on the price, rather than production costs, of a technology.

\subsubsection{Functional Unit and System Boundaries}

As discussed before, the choice of the functional unit has its implications on the validity and usefulness of the derived experience curve, but it should also be aligned with the intended application of the curve. In the context of energy modeling, the curve should ideally be derived for the unit based on which the model makes investment decisions. For many electricity generation technologies, the functional unit would thus most likely be in e.g., EUR/kW of capacity, however, as was discussed before this is not possible or even useful for every technology (see e.g. wind energy).

In terms of system boundaries, it is important to clearly define the technology under investigation, and make decisions on whether to investigate technology components rather than an aggregate technology system. Examples here are PV systems, which have separate learning rates for PV modules vs. inverters, or battery storage systems, which are quite heterogeneous in technological design, as well as being a combination of several components with different potentials for cost reduction, hence will likely have a non-constant learning rate when analyzed as a complete system with a single learning rate.

\subsubsection{Correction for Currency and Inflation}

In order to derive the experience curve, it is necessary that the cost (or price) data is expressed in real terms, e.g., that is corrected for inflation using for instance a GDP-deflator (Junginger et al. 2010; Louwen and Subtil Lacerda 2020), as without this correction, the derived experience curve parameters would otherwise be affected by the rise in prices resulting from inflation. Consequently, datasets with different currencies need to be converted to a single currency.

\subsubsection{Deriving Experience Curve Parameters}

Experience curve parameters can be determined by performing a nonlinear regression of the untransformed data, or by a linear regression of the logarithmically transformed $x$ and $y$ data. Although the discussion on the best approach for experience curves is limited in literature, the consensus seems to be to perform a linear regression of logtransformed data, both within experience curve studies as in the broader scientific literature. 
With the corrected empirical data on prices and cumulative production, the experience curve formula can be fitted by means of a linear regression of the logarithmized data:

$$
\log C=\log C_{1}+b \log N
$$

Using statistical software, the values of parameters $C_{1}$ and $b$ can be derived, including information on the errors of these parameters, their statistical significance, as well as the overall statistical significance of the overall regression. Often a coefficient of determination $\left(R^{2}\right)$ is presented to show the accuracy of the fitted model, although this coefficient has its fair share of critique. Given that statistical software can also provide other goodness of fit metrics, it is recommended to also show these metrics in addition to the $R^{2}$ value.

When performing extrapolations based on the derived experience curves, it is recommended to consider the errors in the learning rate in an uncertainty analysis. Although computation time is likely prohibitive in many modeling applications, it is recommended to take into account the uncertainty of the experience curve parameters explicitly, for instance via a stochastic model formulations using the parameter errors obtained from the regression (van Sark et al. 2010).

\subsection{Experience Curves in Energy System Models}

Within the REFLEX project, one of the main aims was to model pathways toward transformed, low-carbon energy systems up to 2050. As the time horizon increases, the uncertainty regarding technological progress and the associated technology cost reductions becomes larger. Conventional approaches in energy system modeling are to assume an exogenous cost trajectory, uninfluenced by model developments. However, it is unlikely that cost decline occurs over time, without being influenced for e.g., by technology deployment. Therefore, the REFLEX project applied experience curves to be able to model future technology cost developments. Endogenously implemented experience curves allow for an enhanced assessment of the impacts and requirements of policy measures or other incentive schemes. Furthermore, with experience curves, the market diffusion and cost reduction of existing and new energy technologies can be modeled in a feedback loop with cost trajectories derived from experience curves.

In this chapter the technical implementation of experience curves in the various models utilized in the REFLEX project is discussed. Furthermore, several issues related to this model implementation are indicated and possible solutions are examined. 


\subsubsection{Model Implementation of Experience Curves}

In an ideal situation, the derived experience curves are implemented directly in the modeling code, using Eq. (4.2) as shown in Sect. 4.1.1. Implementing this equation directly would allow for endogenous modeling of technology cost development. This is however not feasible for every type of energy system model. Requirements are that the mathematical layout and optimization approach is compatible with implementation of the power law curve of Eq. (4.2), and that the model's geographical scope is global, as technological learning is assumed to occur on a global scale. Alternatively, the implementation can be exogenous, where experience curves are used to calculate cost trajectories using market deployment data from well-aligned (in terms of modeling scenario) global models.

In a direct, endogenous implementation, there is a feedback loop between deployment of technologies and the experience curve. Cumulative technology deployment is given as an input to the experience curve, and the resulting cost is applied in the model in the feedback loop. If the model does not directly produce the required input data (cumulative production), if possible, a conversion from the model outputs to cumulative production can be made, serving as input for the experience curve.

In an exogenous implementation, costs are derived on a time-basis. Market deployment of installed capacities of the respective technologies is taken from an external source, ideally a global energy system or integrated model analyzing a well-aligned modeling scenario. The cumulative technology deployments from this external model are used in conjunction with the experience curve to derive exogenous, time-based cost trajectories, which are fed to the original model.

\subsubsection{Issues with Implementation of Experience Curves in Energy Models}

When implementing experience curves in energy models, a variety of issues can be encountered, such as inadequate geographical scope and technical model constraints. Here, several of these main issues are investigated.

Within the REFLEX energy system modeling framework, most models are limited in geographical scope. Assuming technological learning to occur at a global scale, and thus costs are a function of global cumulative production, this limited geographical scope prevents the models from being able to fully endogenously model costs using the experience curve.

A possible solution here is to derive the cumulative technology deployments for the countries outside the local model from an exogenous global model (Louwen et al. 2018). In this way, there can still be a feedback loop between costs and technology deployment in the local model. However, this means that depending on the geographical scale of the local model, the contribution to global cumulative deployments might be very limited, hence the effect of deployment within the local model 
on cost decline can be small. Furthermore, the global model should be compatible with this approach in terms of geographical subdivision, and should be modeling a well-aligned scenario. An alternative approach would be to determine global capacity developments fully exogenously in terms of small additions from within the local model, using for instance an S-curve approach (Fleiter and Plötz 2013; Schmidt et al. 2017).

The technical design of energy system models is sometimes also inhibitive to implementation of experience curves. Especially optimization models are not compatible with the nonlinear experience curve equation, requiring the implementation of a piece-wise approximation of the function (Barretto 2001; Heuberger et al. 2017). Also, optimization have a so-called 'perfect foresight', meaning technologies with high learning rates are preferred, while contrastingly simulation models avoid technologies with high initial costs, requiring certain incentives to push simulation models to deploy these technologies.

\subsubsection{Description of Energy Models with Implemented Experience Curves}

Within the REFLEX project, experience curves are implemented in the models FORECAST, ELTRAMOD, PowerACE, ASTRA, and TE3 (cf. Chapter 3). The models are linked in what is called an Energy Model System (EMS), where the outputs of some models serve as input for the other models, in several feedback iterations. In this way, the complete European energy system is modeled with high levels of technological detail and including all energy demand and conversion sectors. In the paragraphs below, these models in the context of experience curve implementation are shortly described.

Within the REFLEX project, the FORECAST model is the key model that delivers energy demand figures to the EMS. FORECAST is a bottom-up simulation model with a high level of technological detail, and is primarily aimed to support strategic decisions in the context of long-term development of energy demand and greenhouse gas emissions, and covers the industry, residential and tertiary sectors (Jakob et al. 2012; Fleiter et al. 2018). Within REFLEX, the FORECAST model applied experience curves for heat pumps and alkaline electrolysis (Schreiber et al. 2020 and cf. Chapters 6 and 7), in a semi-endogenous manner, using a combination of technology deployment from within the model and technology deployments from an external integrated assessment model. These two technologies are seen as the most potential appliances for decarbonizing energy demand from buildings and industries.

ELTRAMOD, short for ELectricity TRAnsshipment MODel, is a bottom-up optimization model aiming to model cost-minimal investments and dispatch of electricity generation facilities, including storage and power-to-x technologies (Zöphel et al. 2019; Schreiber et al. 2020). The model covers all EU-27 member states, and additionally includes Norway, Switzerland, United Kingdom, and the Balkan countries. 
Since ELTRAMOD is an optimization model and is not compatible with the nonlinearity of the experience curve function, a procedure was developed to implement experience curves using a piece-wise approximation of the experience curve. However, the implementation of experience curves using this approximation induced significant increases in computation time, hence, given the multi-iterative modeling schedule of the REFLEX EMS, it was opted to include the experience curve data exogenously. In ELTRAMOD, the exogenous experience curves were implemented for carbon capture and storage equipped at a variety of electricity generation technologies, for utility scale battery storage systems (lithium-ion and redox-flow), for heat pumps, and for alkaline electrolysis (cf. Schreiber et al. 2020 and Chapter 10).

PowerACE is a bottom-up and agent-based simulation model that analyzes impacts of market design and policy measures on deployment of low-carbon technologies (cf. Fraunholz et al. 2020 and Chapter 11). Electricity generation companies and other electricity market actors are modeled as individual agents, including electricity demand and generation from renewable resources. The focus of the model is thus the electricity market, supply and demand on a high time-resolution, but investments in generation and storage capacity are also included and are planned in the model on an annual basis (Fraunholz and Keles 2019; Fraunholz et al. 2019). Given the limited geographical scope of the model, experience curves for PowerACE, are implemented exogenously for utility scale electricity storage (lithium-ion and redox-flow batteries) and electricity generation with CCS (Schreiber et al. 2020).

ASsessment of TRAnsport Strategies (ASTRA) is a system dynamics model aimed to provide strategic policy assessment in transport and energy. It covers all EU-27 members, and additionally includes Norway, Switzerland, and the United Kingdom (Fermi et al. 2014 and Chapter 7). ASTRA focuses on passenger and freight transport, including all forms of transport (road, rail, air, and water) and the active modes cycling and walking. It models the development of transport per mode, and can report a variety of indicators like fuel consumption and greenhouse gas emissions. Market diffusion of vehicle technologies is modeled based on a total cost of ownership (TCO) approach that considers among others vehicle prices, fuel or energy prices, and maintenance. In ASTRA, experience curves are implemented for the battery pack component of battery, plug-in hybrid and fuel cell electric vehicles. Although the geographical scope of ASTRA is not global, experience curves are implemented endogenously, as global market developments are supplied by another transport model within the REFLEX EMS, the global model TE3 (Heitel et al. 2020).

The Transport, Energy, Economics, Environment (TE3) model is the final model discussed here. TE3 models passenger road transport, is also a system dynamics model, and is used for policy analysis. It includes all major non-European markets like India, Japan, China, and the United States (Gómez Vilchez and Jochem 2019 and Chapter 5). Technology choice is influenced by several parameters including social dynamics, policy measures, and (available) infrastructure, and technology cost. Like in ASTRA, experience curves are used endogenously in TE3 to model the costs of the battery pack components of battery, plug-in hybrid and fuel cell electric vehicles. 


\subsection{State-of-the-Art Experience Curves and Modeling Results}

Within the REFLEX project, experience curve data was collected for a diverse set of technologies. An overview of the result for selected key technologies is given in Fig. 4.3. For all technologies listed, costs per functional unit are plotted against cumulative installation of each technology in MW.

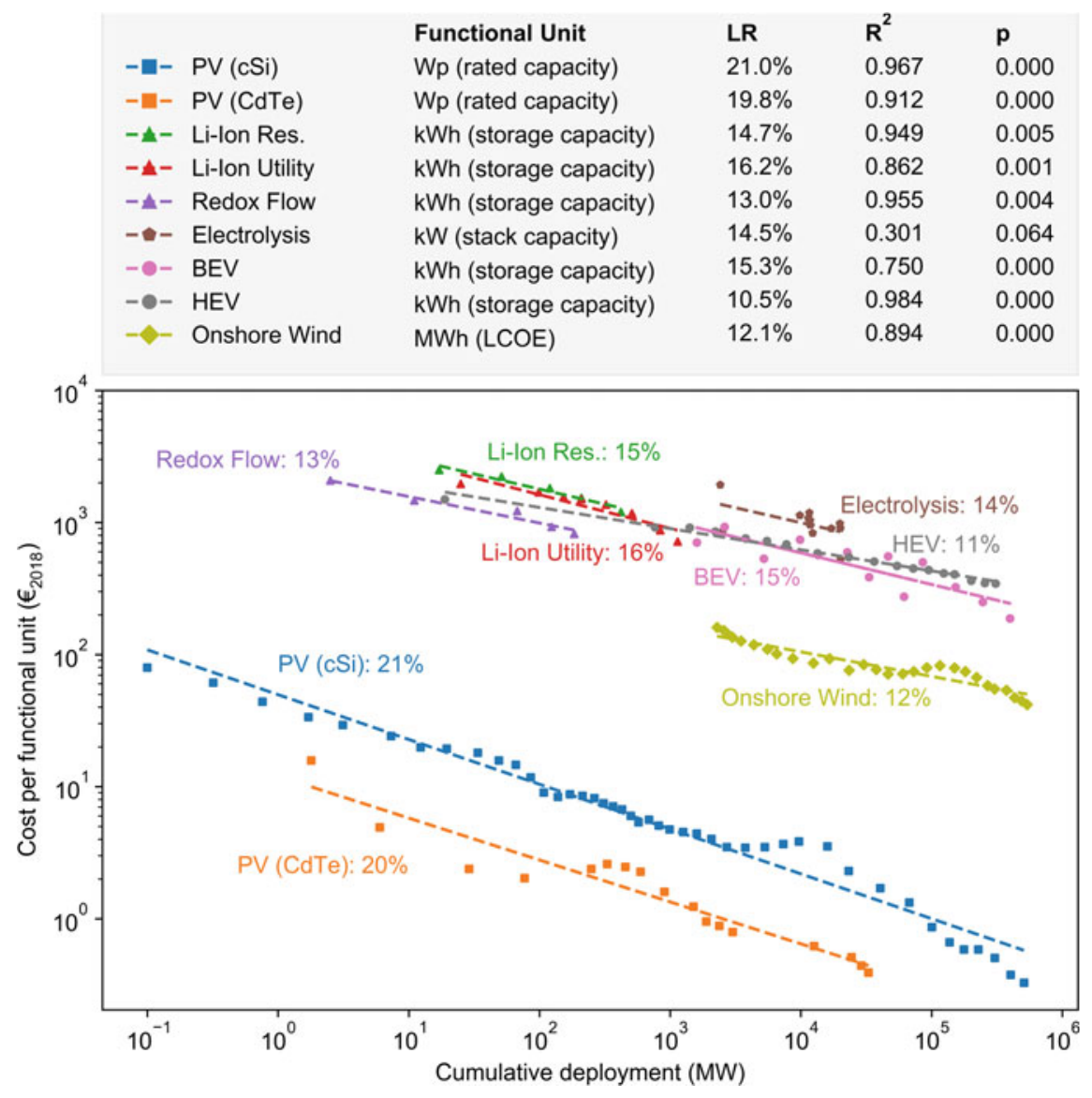

Fig. 4.3 Overview of experience curves for selected technologies. Data sources according to Schmidt et al. (2017), Williams et al. (2017), Louwen et al. (2018), Junginger and Louwen (2020). $\mathrm{LR}=$ learning rate. The $p$-values indicate the $p$-value for the F-test of overall significance of regressions 


\subsubsection{Overview of State-of-the-Art Experience Curves}

For PV modules, both crystalline silicon (cSi) and cadmium telluride (CdTe) based, learning rates of around $20 \%$ are observed, while for other technologies much more varied learning rates were found. Clearly observable in the dataset for PV (cSi) module prices, is a period of price stability, even a small increase in prices around installed capacity of $100 \mathrm{GW}$. This deviation from the overall experience curve trend was the result of a sharp increase in silicon prices, resulting from a shortage in silicon production. Subsequently, when silicon production capacity was greatly expanded and silicon prices declined rapidly, a sharp decline of PV (cSi) module prices occurred.

For heat pumps, learning rates of around $20 \%$ for the total system were observed, based on data from Switzerland, while the learning rate for the specific cost of energy supplied is in the range of $20-25 \%$ due to improvements in the technical performance of heat pumps (Jakob et al. 2020). Although the empirical basis for heat pump experience curves needs to be improved, the high learning rates observed indicate the potential for heat pumps to become cost competitive with natural gas boilers (Jakob et al. 2020).

For alkaline electrolysis, a learning rate of $14.5 \%$ is detected, although the spread of data points results in a low goodness of fit, with a poor $R^{2}$ and a p-value of 0.064 , indicating the regression is not significant at a confidence level of 0.05 . For all other technologies, the regression was found to be significant at this confidence level.

For both utility scale and residential storage systems (respectively, Li-Ion Utility and Li-Ion Res) and for battery electric vehicle (BEV) battery packs, learning rates of around $15 \%$ are observed, while for hybrid electric vehicle (HEV) batteries, the learning rate was found to be much lower, at $11 \%$. For BEV battery packs, significant variance is present in the dataset, leading to a relatively low $R^{2}$ value.

For onshore wind, the data of Williams et al. (2017) are analyzed, which represents levelized cost of electricity (LCOE) for onshore wind systems, in a model that corrects for a variety of parameters, including wind quality of the wind farms that are included in the dataset. A learning rate of $12 \%$ is observed for LCOE as a function of cumulative production, although when the LCOE data is expressed as a function of cumulative electricity generation (rather than cumulative capacity), a higher $R^{2}$ value is obtained, as this also includes a correction for the increase in capacity factors that are observed over time.

The interested reader is referred to Louwen et al. (2018), where detailed results of the project regarding the learning curve approach are presented. 


\subsubsection{Deployments and Cost Developments of Relevant Technologies}

Within the REFLEX project, experience curves have been implemented for a number of technologies. An overview of these technologies is given in Schreiber et al. (2020) and Heitel et al. (2020). In this section the diffusion of key technologies in the different models described above and in Chapter 3 are discussed, and cost trajectories for these technologies are presented.

Transport technologies are modeled within REFLEX with the models ASTRA and TE3. To ensure consistent diffusion of vehicle types, and since the geographical scales of the models are different, market diffusion of transport modes is aligned in the two models in the REFLEX model coupling system (Heitel et al. 2020). The model results (Heitel et al. 2020) indicate that EV battery prices decline to $89 \mathrm{EUR}_{2018} / \mathrm{kWh}$ in 2030 and $59 \mathrm{EUR}_{2018} / \mathrm{kWh}$ in 2050 , down from prices of around $300 \mathrm{EUR}_{2018} / \mathrm{kWh}$ in 2015, the starting year of the transport models, with cumulative global deployment of EV batteries (89\% outside of the EU) approaching $30 \mathrm{TWh}$ in 2050. As expected, the sensitivity analysis shows that especially the uncertainty in the derived learning rates can significantly affect the deployment of EVs. Lower learning rates result in a slower cost decline of vehicle prices, which in turn slows the diffusion of EVs, while with higher learning rates, costs decline faster and market diffusion is also sped up.

Within ELTRAMOD, the optimization model of electricity generation facilities, experience curves are applied exogenously to determine cost developments for battery storage, CCS and power-to-x technologies. As the cost trajectories are thus independent of technology deployment within different modeling scenarios, it is more interesting to discuss sensitivities of these cost trajectories to the applied learning rates. This sensitivity analysis shows that as higher learning rates are assumed for battery storage systems, the deployment of these technologies is increased. This furthermore influences the deployment of other technologies such as CCS and CCGT, which show slightly reduced investments. Similar results are obtained in PowerACE, where impacts of market design and policy measures on deployment of low-carbon technologies are modeled: high learning rates for battery storage systems result in strongly increased deployments of these technologies at the expense of deployments in CCGT and OCGT generation and a strong reduction of deployments of compressed-air energy storage.

Within FORECAST, technological learning is considered for several energy demand technologies. However, since FORECAST is actually a group of models describing different demand sectors separately, an iterative approach was applied to make sure technology deployments in one sector can influence technological learning in the other sectors and vice versa (Schreiber et al. 2020). Heat pumps represent a key technology in the heat demand sector in future energy scenarios, and for this technology a cost reduction of around $20 \%$ was observed up to 2050 , compared to the minimal cost reductions examined without considering technological learning. However, it was found that the installation rates of especially heat demand technologies were less affected by cost developments and technological learning, and 
were to a larger extent the result of scenario constrains (Schreiber et al. 2020). As the technology cost of heat demand technologies only represents a minor share in overall heating systems costs in terms of building refurbishment and the cost differences between different heating technologies are small, energy price differences or the cost for building refurbishment measures have a higher impact on the overall cost structure (Harmsen et al. 2017).

\subsection{Lessons Learned}

As illustrated in this chapter, a comprehensive effort was undertaken to collect data with which state-of-the-art experience curves could be derived and to implement these experience curves in a variety of energy models that are part of the REFLEX Energy Modeling System. Particular effort was devoted to the endogenous implementation of experience curves in the models which was not as straightforward as was anticipated. The main lessons learned during this research are briefly discussed below.

\subsubsection{Methodological Issues}

Regarding general methodological issues, some limitations of the experience curves were identified. These relate to the defined technology system boundaries and functional unit of the experience curve, but also to exogenous parameters affecting cost developments of technologies. Multi-factor experience curves can be a valuable extension of the single-factor experience curves adopted in the REFLEX project, giving the possibility to include the effects of additional parameters such as R\&D activities, commodity prices, and market dynamics. While these multi-factor experience curves could offer the opportunity to attain more accurate cost modeling results, further research is required to make a good comparison between single- and multi-factor experience curves, and to study model implementation of multi-factor experience curves, since model implementation of this type of experience curves is much more complex. Further research should thus investigate the optimal balance between the improved accuracy of results $v s$. the added modeling complexity.

An issue related to system boundaries and functional units is the topic of component-based experience curves. For several technologies evidence are identified that components of the technology system have different learning rates. To accurately represent the cost developments of these technologies on the long term, experience curves for the separate components should be implemented to avoid overestimation of future cost reductions. By using component-based experience curves, the issue of spillover effects across different industries could also be at least partly addressed. Especially for lithium-ion batteries, which are used in electronics, in residential and 
utility scale stationary electricity storage and in electric vehicles, a key recommendation is to investigate experience curves of the different components from battery cells to energy-management systems, although data availability might be prohibitive. As a matter of facts, the model implementation of such component-based experience curves, especially multi-factor version of them, drastically increases the complexity and the requirements of the model to produce input data for the experience curves, making further research on this topic a necessity.

\subsubsection{Model Implementation Issues}

With regard to model implementation, one main issue is how to represent technologies for which there is a lack of empirical data due to their implementation readiness. For early stage technologies like carbon capture and storage (CCS), or energy storage types with small implementation numbers such as compressed-air energy storage, there is almost no empirical data that allows for the derivation of experience curves. This means that the cost developments of these technologies in energy modeling need to be represented either by using experience curves based on proxy technologies, or by some exogenous cost estimations. For high profile technologies like battery-based electricity storage, the available datasets that allow for derivation of experience curves are quite limited in terms of timeframe, hence there is little certainty on the longterm cost developments of these technologies. The implications of this with regard to modeling results should be explicitly discussed, especially in those cases, like the REFLEX project, where the analysis goes up to 30 years ahead. Therefore, a key recommendation is to focus on the creation of cost (and not price) databases that track the costs of these kinds of technologies, for instance by ensuring the assessment and publication of cost data for publicly supported pilot projects for CCS and other novel technologies.

Another theme related to the model implementation of experience curves is the geographical scope of the respective energy model, which is often limited. Assuming technological learning is a global process, some form of exogenous cumulative production data is required. This can be derived through a variety of approaches, such as by using results from external global models that analyze well-aligned scenarios, or alternatively by using the S-curve based approach. Within the REFLEX project, a combination of these measures was applied on a more or less ad hoc basis. Future research projects that aim to implement experience curves in a similar manner in geographically restricted energy models, should outline an explicit approach to deal with this problem, for instance by including a global energy or integrated assessment model in the modeling activities, ensuring a consistent scenario analysis across global and regional models.

There were also several technical issues identified with regard to model implementation. A typical problem is the fact that the power curve function of experience curves is not compatible with some models, requiring the implementation of a piecewise linear approximation of the original function. Also, models tend to have either 
perfect foresight (optimization models) or contrastingly, a myopic view (simulation models), which could result in over- or underrepresentation of technologies in the modeling outcomes. Finally, it was found in the REFLEX project that the modeling scenarios that are investigated can also be a barrier for the implementation of experience curves for certain technologies; this is the case where technology deployments are defined as scenarios inputs, for instance by means of targets for the deployment of renewable energy technologies.

\subsection{Conclusions}

A final recommendation is to further develop research studies on applications of experience curve and technological learning mechanisms outside the economy sector. As part of the REFLEX project scientific literature review, several studies were identified that use experience curve to describe developments in energy efficiency of appliances and industrial processes (Jakob and Madlener 2004; Ramírez and Worrell 2006; Weiss et al. 2010; Brucker et al. 2014; Fleiter et al. 2017) as well as greenhouse gas and energy demand of renewable energy technologies (Bergesen and Suh 2016; Louwen et al. 2016). Furthermore, the concept of technological learning has been applied in the domain of social sciences to represent the effects of social learning on the market diffusion of battery electric vehicles (Edelenbosch et al. 2018). It would be therefore extremely interesting to expand the scope of application of experience curves in energy modeling so as to allow for the endogenous modeling of these additional parameters.

\section{References}

Barretto L (2001) Technological learning in energy optimization models and deployment of emerging technologies. Swiss Federal Institute of Technology, Zurich, Switzerland.

Bergesen JD, Suh S (2016) A framework for technological learning in the supply chain: a case study on CdTe photovoltaics. Appl Energy 169:721-728. https://doi.org/10.1016/J.APENERGY.2016. 02.013

Boston Consulting Group (1970) Perspectives on experience. Boston Consulting Group

Brucker N, Fleiter T, Plötz P (2014) What about the long term? Using experience curves to describe the energy-efficiency improvement for selected energy-intensive products in Germany. In: ECEEE industrial summer study proceedings. Arnhem, The Netherlands: eceee, pp 341-352

Edelenbosch OY, McCollum DL, Pettifor H, Wilson C, van Vuuren DP (2018) Interactions between social learning and technological learning in electric vehicle futures. Environmental Research Letters. IOP Publishing, 13(12):124004. https://doi.org/10.1088/1748-9326/aae948

Fermi F, Fiorello D, Krail M, Schade W (2014) Description of the ASTRA-EC model and of the user interface. Deliverable D4.2 of ASSIST (Assessing the social and economic impacts of past and future sustainable transport policy in Europe). Project co-funded by European Commission 7thRTD Programme. Karlsruhe, Germany 
Fleiter T, Elsland R, Herbst A, Manz P, Popovski E, Rehfeldt M, Reiter U, Catenazzi G, Jakob M, Harmsen R, Rutten C, Dittmann F, Rivière P, Stabat P (2017) Baseline scenario of the heating and cooling demand in buildings and industry in the 14 MSs until 2050. Karlsruhe

Fleiter T, Rehfeldt M, Herbst A, Elsland R, Klingler AL, Manz P, Eidelloth S (2018) A methodology for bottom-up modelling of energy transitions in the industry sector: The FORECAST model. Energy Strat Rev 22:237-254. https://doi.org/10.1016/j.esr.2018.09.005

Fleiter T, Plötz P (2013) Diffusion of energy-efficient technologies. In: Encyclopedia of energy, natural resource, and environmental economics, Elsevier, pp 63-73. https://doi.org/10.1016/b9780-12-375067-9.00059-0

Fraunholz C, Hladik D, Keles D, Möst D, Fichtner W (2020) On the long-term efficiency of market splitting in Germany. Working paper series in Production and Energy, 38. https://doi.org/10.5445/ ir/1000105902

Fraunholz C, Keles D (2019) Report on investments in flexibility options considering different market designs. REFLEX project report

Fraunholz C, Keles D, Fichtner W (2019) On the role of electricity storage in capacity remuneration mechanisms. Working Paper Series in Production and Energy, 37. https://doi.org/10.5445/ir/100 0104471

Gómez Vilchez JJ, Jochem P (2019) Simulating vehicle fleet composition: a review of system dynamics models. Renew Sustain Energy Rev 115:109367. https://doi.org/10.1016/j.rser.2019. 109367

Harmsen R, van Zuijlen B, Fleiter T, Manz P, Elsland R, Reiter U, Catenazzi G, Jakob M (2017) Cost-curves for heating and cooling demand reduction in the built environment and industry

Heitel S, Seddig K, Gómez Vilchez JJ, Jochem P (2020) Global electric car market deployment considering endogenous battery price development. In: Junginger M, Louwen A (eds) Technological learning in the transition to a low-carbon energy system, Elsevier, pp 281-305. https:// doi.org/10.1016/b978-0-12-818762-3.00015-7

Heuberger CF, Rubin ES, Staffell I, Shah N, Mac Dowell N (2017) Power capacity expansion planning considering endogenous technology cost learning. Appl Energy 204: 831-845. https:// doi.org/10.1016/j.apenergy.2017.07.075

Jakob M, Fleiter T, Catenazzi G, Hirzel S, Reitze F, Toro F (2012) The impact of policy measures on the electricity demand of the tertiary sector of the European countries: an analysis with the bottomup model FORECAST. In: Improving energy efficiency in commercial buildings conference

Jakob M, Reiter U, Krishnan S, Louwen A, Junginger M (2020) Heating and cooling in the built environment. In: Technological learning in the transition to a low-carbon energy system, Elsevier, pp 189-219. https://doi.org/10.1016/b978-0-12-818762-3.00011-x

Jakob M, Madlener R (2004) Riding down the experience curve for energy-efficient building envelopes: the Swiss case for 1970-2020. Int J Energy Technol Policy 2(1-2): 153-178

Junginger M, van Sark W, Faaij A (2010) Technological learning in the energy sector. Edward Elgar Publishing. https://doi.org/10.4337/9781849806848

Junginger M, Louwen A (eds) (2020) Technological learning in the transition to a low-carbon energy system, technological learning in the transition to a low-carbon energy system, Elsevier. https:// doi.org/10.1016/c2018-0-04547-8

Kittner N, Lill F, Kammen DM (2017) Energy storage deployment and innovation for the clean energy transition. Nature Energy 2(9):17125. https://doi.org/10.1038/nenergy.2017.125

Louwen A, van Sark W, Faaij A, Schropp R (2016) Re-assessment of net energy production and greenhouse gas emissions avoidance after 40 years of photovoltaics development. Nature Communications, 7. https://doi.org/10.1038/ncomms13728

Louwen A, Junginger M, Krishnan AL (2018) Technological learning in energy modelling: experience curves. Policy Brief for REFLEX project

Louwen A, Subtil Lacerda J (2020) The experience curve: concept, history, methods, and issues. In Junginger $\mathrm{M}$ and Louwen A (eds) Technological learning in the transition to a low-carbon energy system, Elsevier, pp 9-31. https://doi.org/10.1016/b978-0-12-818762-3.00002-9 
Nemet GF (2009) Interim monitoring of cost dynamics for publicly supported energy technologies. Energy Policy 37(3), pp 825-835. https://doi.org/10.1016/J.ENPOL.2008.10.031

Ramírez CA, Worrell E (2006) Feeding fossil fuels to the soil: an analysis of energy embedded and technological learning in the fertilizer industry. Resour, Conserv Recycl 46(1):75-93. https://doi. org/10.1016/J.RESCONREC.2005.06.004

van Sark W, Nemet GF, Kahouli-Brahmi S, Neij L, Wene CO (2010) General aspects and caveats of experience curve analysis. In: Technological learning in the energy sector: lessons for policy, industry and science, pp 18-35

Schmidt O, Hawkes A, Gambhir A, Staffell I (2017) The future cost of electrical energy storage based on experience rates. Nat Energy 2(8). https://doi.org/10.1038/nenergy.2017.110

Schreiber S, Zöphel C, Fraunholz C, Reiter U, Herbst A, Fleiter T, Möst D (2020) Experience curves in energy models-lessons learned from the REFLEX project. In: Junginger M and Louwen A (eds) Technological learning in the transition to a low-carbon energy system, Elsevier, pp 259-279. https://doi.org/10.1016/b978-0-12-818762-3.00014-5

SolarPower Europe (2019) Global market outlook for solar power 2019-2023

Weiss M, Patel MK, Junginger M, Blok K (2010) Analyzing price and efficiency dynamics of large appliances with the experience curve approach. Energy Policy. https://doi.org/10.1016/j.enpol. 2009.10.022

Williams E, Hittinger E, Carvalho R, Williams R (2017) Wind power costs expected to decrease due to technological progress. Energy Policy 106:427-435. https://doi.org/10.1016/J.ENPOL.2017. 03.032

Wright TP (1936) Factors affecting the cost of airplanes. J Aeronaut Sci 3(February):122-128

Yeh S, Rubin ES (2012) A review of uncertainties in technology experience curves. Energy Econ 34(3):762-771. https://doi.org/10.1016/J.ENECO.2011.11.006

Zöphel C, Schreiber S, Herbst A, Klingler AL, Manz P, Heitel S, Fermi F, Wyrwa A, Raczyński M, Reiter U (2019) Report on cost optimal energy technology portfolios for system flexibility in the sectors heat, electricity and mobility. REFLEX project report

Open Access This chapter is licensed under the terms of the Creative Commons Attribution 4.0 International License (http://creativecommons.org/licenses/by/4.0/), which permits use, sharing, adaptation, distribution and reproduction in any medium or format, as long as you give appropriate credit to the original author(s) and the source, provide a link to the Creative Commons license and indicate if changes were made.

The images or other third party material in this chapter are included in the chapter's Creative Commons license, unless indicated otherwise in a credit line to the material. If material is not included in the chapter's Creative Commons license and your intended use is not permitted by statutory regulation or exceeds the permitted use, you will need to obtain permission directly from the copyright holder.

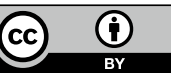

